\title{
Structural properties and phase transitions in a silica clathrate
}

\author{
$\operatorname{AUTHOR}(\mathrm{S}):$ \\ Y. Liang; F. O. Ogundare; C. R. Miranda; J. K. \\ Christie; S. Scandolo
}

\section{CITATION:}

Y. Liang ... [et al]. Structural properties and phase transitions in a silica clathrate. JOURNAL OF CHEMICAL PHYSICS 2011, 134(7): 074506.

\section{ISSUE DATE:}

2011-02

URL:

http://hdl.handle.net/2433/160659

\section{RIGHT:}

Copyright 2011 American Institute of Physics. This article may be downloaded for personal use only. Any other use requires prior permission of the author and the American Institute of Physics. The following article appeared in JOURNAL OF CHEMICAL PHYSICS 134, 074506 (2011) and may be found at http://link.aip.org/link/?jcp/134/074506 


\section{AIP $\begin{aligned} & \text { mesolumalot } \\ & \text { Chemical Physics }\end{aligned}$}

\section{Structural properties and phase transitions in a silica clathrate}

Yunfeng Liang, Folorunso O. Ogundare, Caetano R. Miranda, Jamieson K. Christie, and Sandro Scandolo

Citation: J. Chem. Phys. 134, 074506 (2011); doi: 10.1063/1.3532543

View online: http://dx.doi.org/10.1063/1.3532543

View Table of Contents: http://jcp.aip.org/resource/1/JCPSA6/v134/i7

Published by the American Institute of Physics.

\section{Additional information on J. Chem. Phys.}

Journal Homepage: http://jcp.aip.org/

Journal Information: http://jcp.aip.org/about/about_the_journal

Top downloads: http://jcp.aip.org/features/most_downloaded

Information for Authors: http://jcp.aip.org/authors

\section{ADVERTISEMENT}

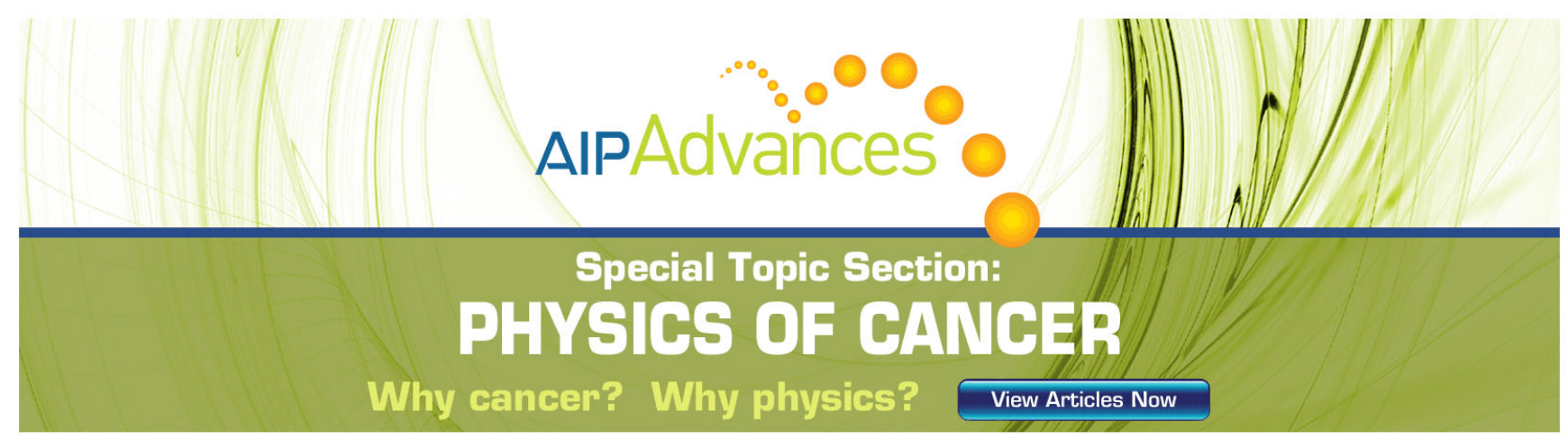




\title{
Structural properties and phase transitions in a silica clathrate
}

\author{
Yunfeng Liang, ${ }^{1,2, a)}$ Folorunso O. Ogundare, ${ }^{3,4}$ Caetano R. Miranda, ${ }^{2,3,5}$ \\ Jamieson K. Christie, ${ }^{2,3, b)}$ and Sandro Scandolo ${ }^{2,3}$ \\ ${ }^{1}$ International School for Advanced Studies (SISSA), Via Beirut 2-4, 34014 Trieste, Italy \\ ${ }^{2}$ INFM/Democritos National Simulation Center, 34014 Trieste, Italy \\ ${ }^{3}$ The Abdus Salam International Centre for Theoretical Physics (ICTP), Strada Costiera 11, \\ 34014 Trieste, Italy \\ ${ }^{4}$ Department of Physics, University of Ibadan, Ibadan, Nigeria \\ ${ }^{5}$ Universidade Federal do ABC, Rua Santa Adélia, 166 Bangu 09210-170, Santo André, SP, Brazil
}

(Received 6 September 2010; accepted 6 December 2010; published online 18 February 2011)

\begin{abstract}
Melanophlogite, a low-pressure silica polymorph, has been extensively studied at different temperatures and pressures by molecular dynamics simulations. While the high-temperature form is confirmed as cubic, the low-temperature phase is found to be slightly distorted, in agreement with experiments. With increasing pressure, the crystalline character is gradually lost. At $8 \mathrm{GPa}$, the radial distribution function is consistent with an amorphous state. Like pristine glass, the topology changes, plastic behavior, and permanent densification appear above $\sim 12 \mathrm{GPa}$, triggered by Si coordination number changes. We predict that a partial crystalline and amorphous sample can be obtained by recovering the sample from a pressure of $\sim 12-16 \mathrm{GPa}$. (C) 2011 American Institute of Physics. [doi:10.1063/1.3532543]
\end{abstract}

\section{INTRODUCTION}

The crystalline-to-amorphous transformation under high pressure and low temperature is currently the subject of intense study, since the discovery of amorphization of $\mathrm{H}_{2} \mathrm{O}$ ice $\mathrm{I}_{\mathrm{h}}$ under pressure. ${ }^{1}$ Interest has focused on the possible occurrence of pressure-induced amorphization in silica polymorphs, ${ }^{2}$ the thermodynamic and mechanistic basis of the process, and the relationship between amorphization by pressure and by other means. ${ }^{3,4}$ Amorphization of minerals (e.g., silica and silicates) is observed in situ with a variety of structural probes such as Raman and IR spectroscopy and $\mathrm{x}$-ray diffraction on samples usually compressed in diamond anvil cells. ${ }^{3,4}$ One assumes amorphization when diffraction peaks vanish or when broad, glasslike bands appear in vibrational spectra. The detailed microstructural origins are however rarely extracted from experiments alone.

Recent work on zeolite collapse has renewed interest in pressure-induced amorphization, ${ }^{5-7}$ where crystalline order can be preserved to some extent in the densified sample. In particular, Greaves et al. have reported that zeolite $\mathrm{Y}$ was thermally amorphized $20 \%$ at $650{ }^{\circ} \mathrm{C}, 60 \%$ at $750{ }^{\circ} \mathrm{C}$, and $100 \%$ at $850{ }^{\circ} \mathrm{C}$. A $90 \%$ amorphized specimen was fabricated in a multianvil press at $8 \mathrm{GPa}$ and room temperature. The degree of amorphization was initially obtained by comparing the intensity of $\mathrm{x}$-ray diffraction powder patterns with that of the starting materials ${ }^{5}$ and then further identified by the boson peaks. ${ }^{6}$ Further zero-temperature calculations have theoretically shown that the different degrees of amorphization

\footnotetext{
a) Author to whom correspondence should be addressed. Electronic mail: y_liang@earth.kumst.kyoto-u.ac.jp. Present address: Department of Urban Management, Kyoto University, Kyoto, 615-8540, Japan.

b) Present address: Department of Chemistry, University College London, 20 Gordon Street, London WC1H 0AJ, United Kingdom.
}

in zeolites A and $\mathrm{Y}$ are coincident with different first-order transitions toward the high-density amorphous phase. ${ }^{7}$ Very recently, an amorphous silicate phase was obtained by pressurizing the zeolite silicalite-1-F, and reverse Monte Carlo (RMC) modeling suggested that its structure was consistent with a topologically ordered phase. ${ }^{8}$ However, there are possible ambiguities in using RMC to interpret these experimental findings. In particular, RMC can only produce a structure which is consistent with the fitted experimental data; since there are very many such possible structures for any given set of data, RMC cannot be said to be predictive. A more convincing picture of the microscopic mechanisms of the phase transition under pressure, in particular, whether a densification can happen without topological change (i.e., bond breaking and reforming), could be obtained by direct observation of the atomic scale processes responsible for the phase transition. Such an "experiment" can be more easily carried out with molecular dynamics (MD) simulations than with other simulation techniques. Therefore we have studied the highpressure behavior of a $\mathrm{SiO}_{2}$ clathrate called melanophlogite, which is an aluminum-free prototype of zeolite, ${ }^{9-25}$ by using molecular dynamics simulations. The high quality of the recently developed $\mathrm{SiO}_{2}$ force field ${ }^{26}$ used gives us confidence that the results of this study are accurate.

Melanophlogite (type $\mathrm{I} \mathrm{SiO}_{2}$ clathrate) is a rare mineral first described by Lasulx in $1876,{ }^{11}$ known from only five volcanic areas. The name melanophlogite describes the fact that the mineral turns black when heated. Melanophlogite contains up to $8 \% \mathrm{C}, \mathrm{H}, \mathrm{O}, \mathrm{N}$, and $\mathrm{S}^{12}$ The role of these additional elements was not understood until Kamb concluded from x-ray powder data that melanophlogite is isostructural with the cubic gas hydrates of type I (Fig. 1), ${ }^{13}$ in which there are two types of cage per unit cell: two pentagonal dodecahedra $\left[5^{12}\right]$ and six tetrakaidecahedra $\left[5^{12} 6^{2}\right]$ cages 


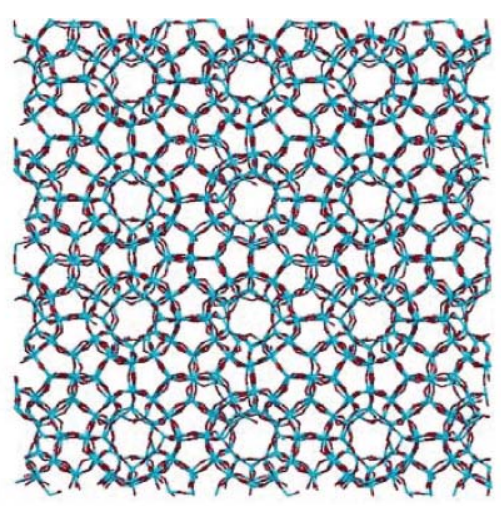

(a)

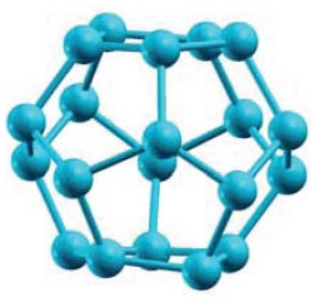

(b)

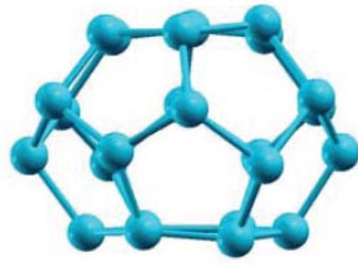

(c)
FIG. 1. A snapshot at $1000 \mathrm{~K}$ of the melanophlogite structure (a), of one of the pentagonal dodecahedral $\left[5^{12}\right]$ cages $(b)$, and of one of the tetrakaidecahedral $\left[5^{12} 6^{2}\right]$ cages (c).

with the guest molecules $\mathrm{M}^{12}\left(\mathrm{~N}_{2}, \mathrm{CH}_{4}\right)$ and $\mathrm{M}^{14}\left(\mathrm{CO}_{2}\right)$, respectively. ${ }^{11-13}$ The guest molecules are thought to act as templates during crystallization. Using a mixture of $\mathrm{CO}_{2}$, $\mathrm{CH}_{4}$, and $\mathrm{N}_{2}$ as guest molecules, melanophlogite was first synthesized under hydrothermal conditions from aqueous silica solutions in $1982 .{ }^{14}$ Melanophlogite is known to distort slightly from cubic symmetry below around $80{ }^{\circ} \mathrm{C} .{ }^{15-22}$ By careful thermal treatment (e.g., heating to above $600^{\circ} \mathrm{C}$ ), the guest molecules can be removed..$^{9,10,12,18-22}$ In the past, many studies have been performed in order to understand the guest molecules trapped in the clathrates ${ }^{23,24}$ and the role of the guest for the response of the host framework to pressure. ${ }^{25}$ More recently, crystalline-amorphous transitions have been documented in guest-free melanophlogite through a broadening and drop in the intensity of the x-ray diffraction lines at $\sim 8 \mathrm{GPa} .{ }^{9}, 10$ The microstructure information is however still lacking: particularly how the different cages respond to the pressure, whether the partial crystalline structure exists in densified melanophlogite, and whether the topology of the network is preserved as has been suggested in guest-free silicalite. $^{8}$

In this paper, we present extensive molecular dynamics simulations of guest-free melanophlogite at different temperatures and pressures. Our main findings are that (1) the temperature-induced phase transition can be explained using the same mechanism underlying the $\alpha-\beta$ phase transitions in quartz and cristobalite and the zero thermal expansion at high temperature can be explained solely in terms of expansion of the individual cages; (2) the x-ray amorphous material obtained by compressing melanophlogite between 8 and $12 \mathrm{GPa}$ is topologically identical to the crystalline phase; (3) parts of the crystalline structure still exist in densified melanophlogite subjected to pressures of $\sim 12-16 \mathrm{GPa}$.

The paper is organized as follows: in Sec. II, we describe the methodology; in Sec. III, we first present the structural changes with temperature, across the $\alpha-\beta$ phase transition. There we show that it is possible to analyze the phase transformations by the distortion of the cages. We then present the changes with pressure of the equation of state, $\mathrm{x}$-ray structure factor, radial distribution functions, and the structural changes of the two different cages. In the last part of Sec. III, we discuss the structural properties of the densified sample.

\section{METHODOLOGY}

In this paper, we present the results of simulations of the pressure-induced amorphization of guest-free melanophlogite. The simulations were carried out using an interatomic force field optimized by best fit on first-principles (densityfunctional theory) calculations. ${ }^{26}$ The force field describes the structural and vibrational properties of $\mathrm{most}^{\mathrm{SiO}_{2}}$ crystalline polymorphs, liquid, and glass ${ }^{27-31}$ better than all the available force fields to which it has been compared so far. In particular, it describes the thermodynamic stability of the crystalline polymorphs of silica, ${ }^{31}$ including the pressure dependence of the lattice constant and the phonon softening across the rutileto- $\mathrm{CaCl}_{2}$ transition. ${ }^{27,28}$ We start our MD simulations from a cell consisting of $368 \mathrm{SiO}_{2}$ formula units $(2 \times 2 \times 2$ unit cells $)$, which is obtained by first inserting oxygen atoms in the middle of the line connecting two silicon atoms in type I silicon clathrate and then relaxing the structure to $0 \mathrm{GPa}$ and $0 \mathrm{~K}$. The

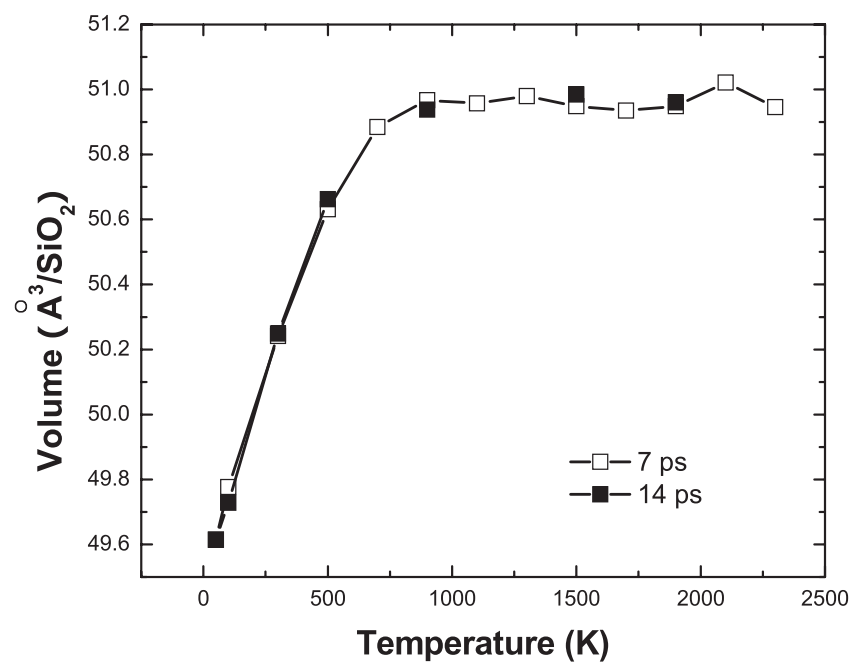

FIG. 2. Volume as a function of temperature, averaged over the initial $7 \mathrm{ps}$ of the simulation (open symbols) and over the subsequent $7 \mathrm{ps}$ (solid symbols). The difference between the two results provides an estimate of the statistical error due to the finite simulation time. 

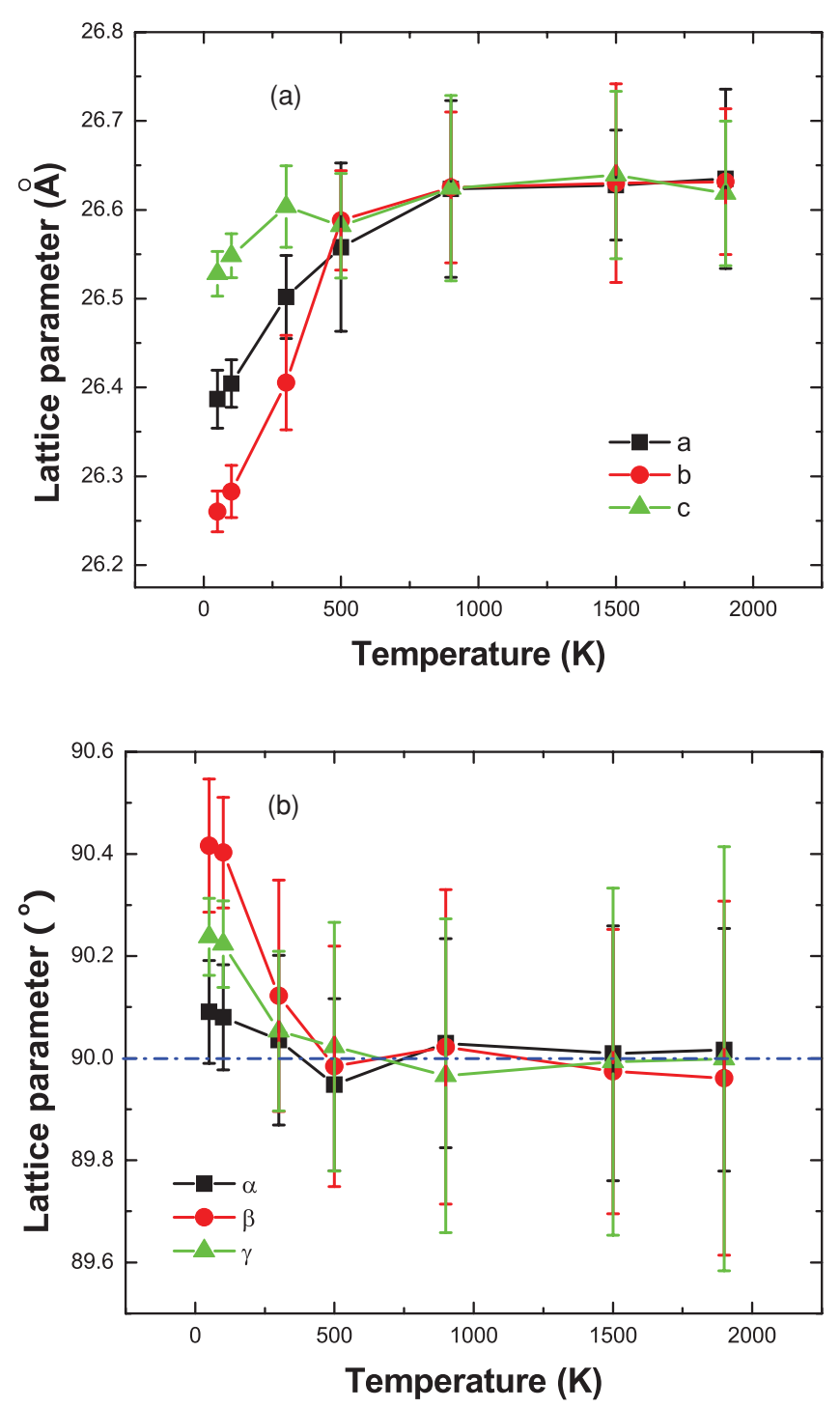

FIG. 3. Lattice parameters: lengths (a) and angles (b) as a function of temperature obtained as an average over the last $7 \mathrm{ps}$ of the simulation.

initial velocity was set randomly according to the Maxwell distribution. The MD time step was set to 0.72 fs (30 a.u.).

In order to monitor the structural changes (shape and size) of the two cage types $\left(\left[5^{12}\right]\right.$ and $\left[5^{12} 6^{2}\right]$ ) across the transitions induced either by temperature or by pressure, we calculated radial distribution functions between atoms and imaginary points located at the crystallographic center of the cages. We analyzed radial distribution functions using a method introduced recently by Saitta and co-workers to study the short- and intermediate-range order changes of liquid water ${ }^{32}$ and amorphous ices. ${ }^{33,34}$ They ranked the neighbors (to any tagged one) in order of increasing distance and analyzed the (individual or combined) neighbor distance distributions. From this analysis, they drew insights on the structural changes in liquid and amorphous water (ice) in terms of first (short range) and second neighbor shells (intermediate range). By ranking the $\mathrm{Si}$ neighbors to the cage center in the $\left[5^{12}\right]$ cages (cage 1 ) and taking the first 20 neighbors, we then extract the neighbor list constructed for cage 1 only. Similarly, by ranking the Si neighbors to the cage center in the $\left[5^{12} 6^{2}\right]$
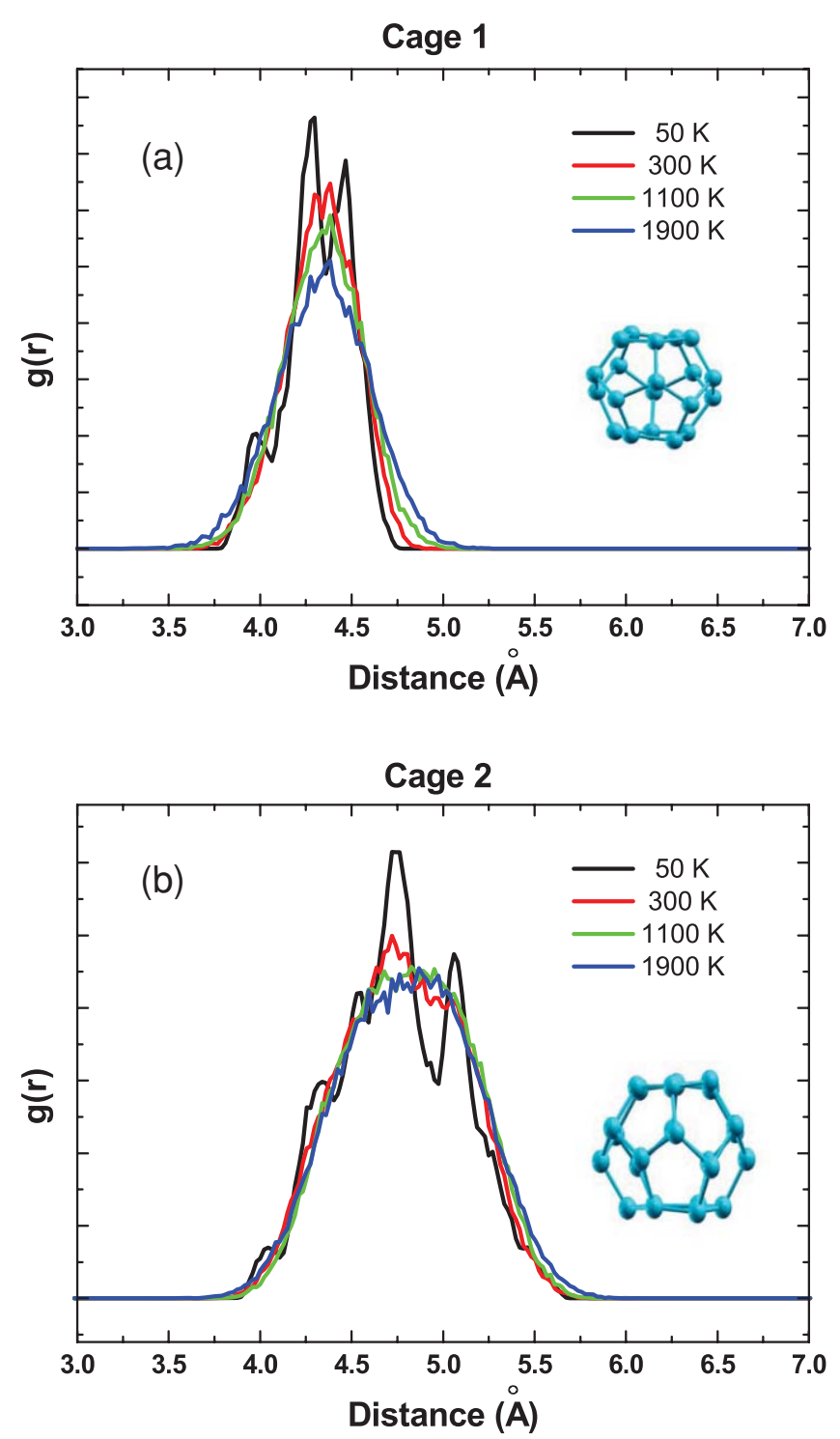

FIG. 4. Radial distribution functions of $\mathrm{Si}$ atoms with respect to the central point of the cages at four different temperatures: for pentagonal dodecahedra $\left[5^{12}\right]$ cage (a) and tetrakaidecahedra $\left[5^{12} 6^{2}\right]$ cage (b).

cages (cage 2) and taking the first 24 neighbors, we then extract the neighbor list constructed for cage 2 only. We then analyze the distance distribution function with respect to the cage centers to get some insights into the distortion of the two different cages under different temperatures or pressures.

\section{RESULTS AND DISCUSSIONS}

\section{A. Temperature-induced symmetrization}

We first studied the lattice and volume changes at different temperatures (Figs. 2 and 3). It is interesting to note that the volume expands very fast with increasing temperature and then saturates at around $700 \mathrm{~K}$, which is typical of the $\alpha-\beta$ transition such as that in quartz and cristobalite. ${ }^{27,28,35}$ Like quartz and cristobalite, we find that melanophlogite also shows the dynamic disorder of oxygen atoms above $\sim 500 \mathrm{~K}$. While the high-temperature form is found to be cubic, in agreement with experiments, the low-temperature phase is 


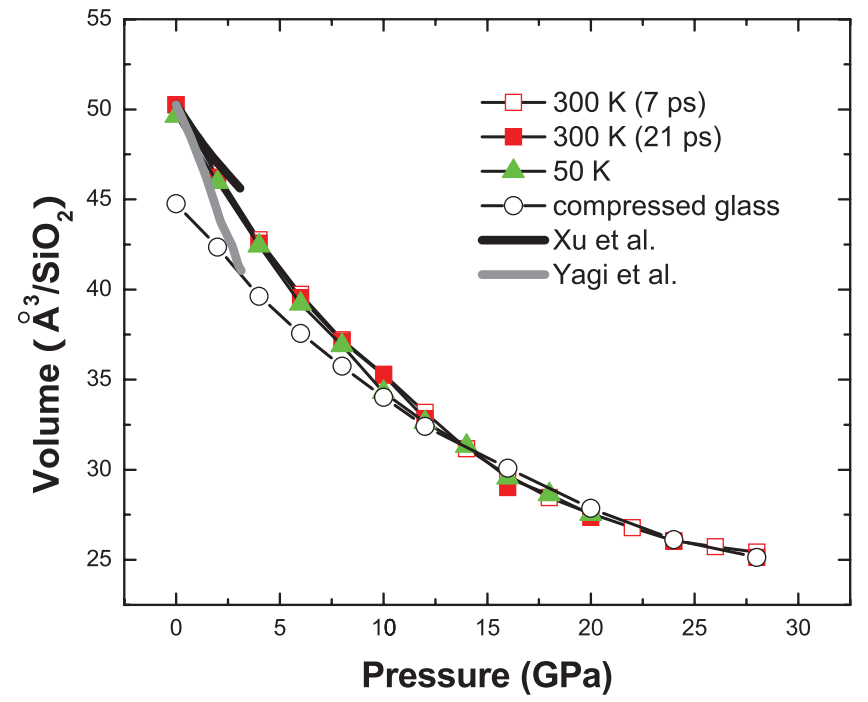

FIG. 5. Equation of state of melanophlogite at $300 \mathrm{~K}$, averaged over the first $7 \mathrm{ps}$ (open squares) and over the subsequent $14 \mathrm{ps}$ (solid squares). The equation of state at $50 \mathrm{~K}$ (triangles) is already converged after the initial few picoseconds. Data for cold compressed glass (circles) obtained with the same potential employed here (Ref. 28) and experimental data (bold line) (Refs. 8 and 9) are also reported for comparison.

found to be slightly distorted (Fig. 3), which is also in agreement with experiments. ${ }^{10,15-22}$ The experimental studies have found the low-temperature phase as tetragonal for guest-bearing melanophlogite, whereas a lower symmetry (probably, an orthorhombic phase) was reported for guestfree melanophlogite. ${ }^{18}$ Here, we found that the original cell was not even orthogonal at temperatures below $500 \mathrm{~K}$ (see Fig. 3). The calculated lattice parameter length differences at $300 \mathrm{~K}$ are within $0.3 \%$, which is again in good agreement with the experiments. ${ }^{10,15-22}$ However, such small differences (e.g., $0.1^{\mathrm{o}}$ in angles or $0.3 \%$ in lengths at $300 \mathrm{~K}$ ) can hardly be resolved by $\mathrm{x}$-ray diffraction patterns as discussed in Ref. 18.

We then investigated the changes of the two different cages by ranking the $\mathrm{Si}$ neighbors to the cage centers and calculating the distance distribution functions. At room temperature, the distribution for cage 1 is well peaked at $4.38 \AA$ (shown in Fig. 4), and for cage 2 it is peaked at $4.87 \AA$ with a shoulder at $\sim 5.0 \AA$. By taking account of the covalent radius of $\mathrm{Si}$ atoms, the dimensions of the cages are similar to the values estimated by Gies. ${ }^{16}$ As shown in Fig. 4, cages 1 and 2 in the $\beta$-phase ( $>700 \mathrm{~K}$ ) do not expand with increasing temperature. In particular, the sizes are similar to each other for the samples at 1100 and $1900 \mathrm{~K}$. Since melanophlogite is exclusively made up of two types of the cages, a pentagonal dodecahedra $\left[5^{12}\right]$ cage and a tetrakaidecahedra $\left[5^{12} 6^{2}\right]$ cage (as shown in Figs. 1(b) and 1(c)), the invariance of the volume of the individual cages in the high-temperature regime is consistent with the zero thermal expansion of the crystal. Similarly, the symmetry distortion of the lattice at low temperature can be understood by analyzing the distortion of the cages. The radial function in cage 1 at $50 \mathrm{~K}$ is split into two peaks at 4.30 and $4.47 \AA$, respectively. In cage 2 , the two peaks are located at 4.75 and $5.06 \AA$. The distortions are as large as 0.31 $\AA$ in cage 2 and $0.17 \AA$ in cage 1 , respectively. Symmetry
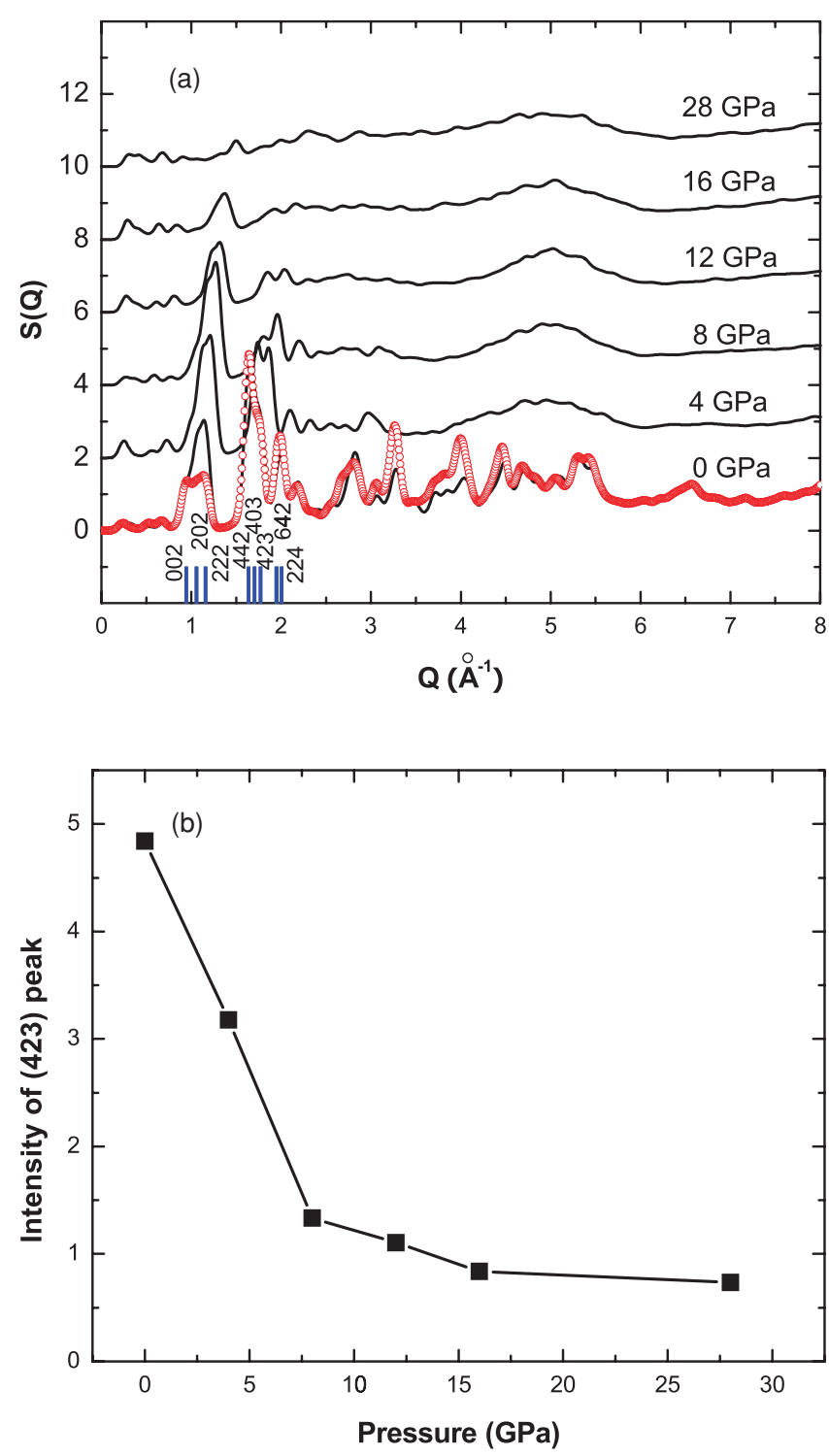

FIG. 6. (a) Calculated $\mathrm{x}$-ray structure factor at some selected pressures at $300 \mathrm{~K}$ (line) and $900 \mathrm{~K}$ (circle), and (b) intensity of Bragg peak [423] ( 1.75 $\AA^{-1}$ ) as a function of pressure.

distortions of the order of $0.1-0.3 \AA$ for the cages, which become less spherical at low temperature, are consistent with the calculated low-temperature distortions of the lattice parameters.

\section{B. Pressure-induced amorphization}

Next, we applied a stepwise hydrostatic compression at 300 and $50 \mathrm{~K}$, in pressure increments of $2 \mathrm{GPa}$, followed, at each pressure, by an equilibration time of $20 \mathrm{ps}$. No clear volume collapse can be identified in the equation of state, up to a pressure of $28 \mathrm{GPa}$ (Fig. 5). Melanophlogite has higher compressibility than glass and the equation of state at lower pressure lies in between two recent experiments, ${ }^{9,10}$ while the unit volume converges to pristine glass at pressures above $12 \mathrm{GPa}$. No sharp volume collapse has been found in the whole studied range. We compared the x-ray structure factor at different pressures in Fig. 6. At $0 \mathrm{GPa}$ and room temperature, 

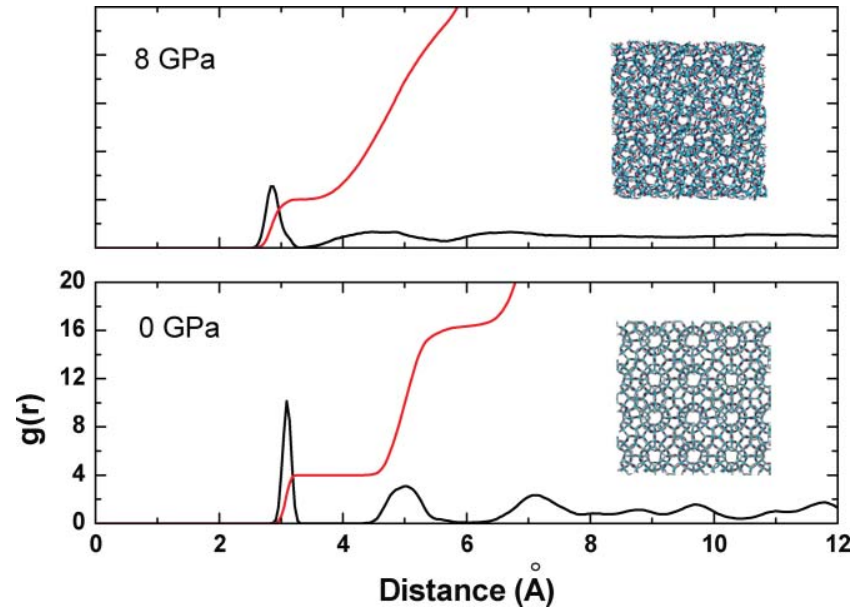

FIG. 7. Si-Si radial distribution functions at 0 and $8 \mathrm{GPa}$.

Cage 1

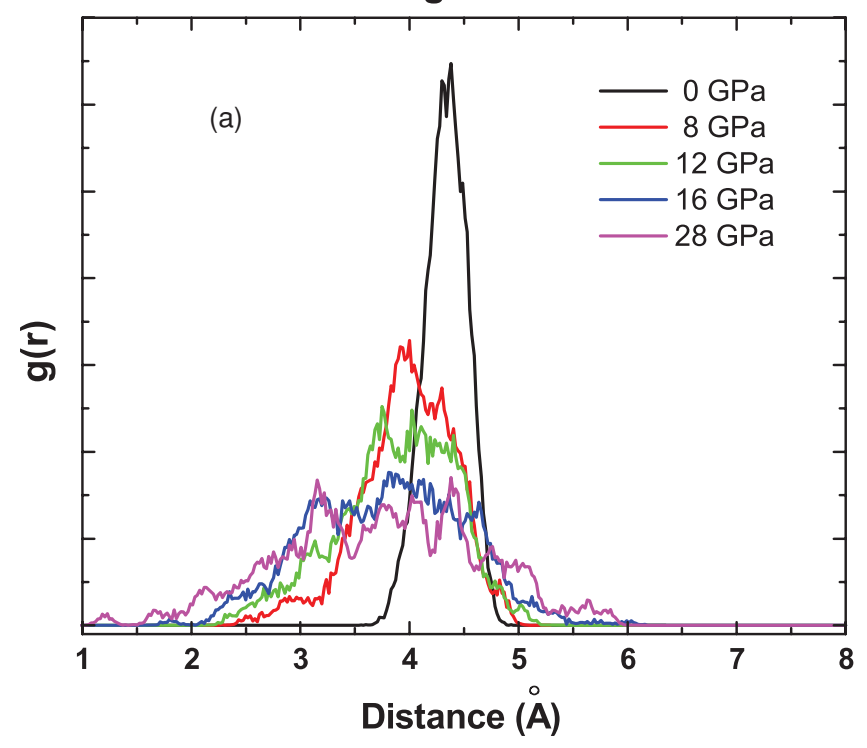

Cage 2

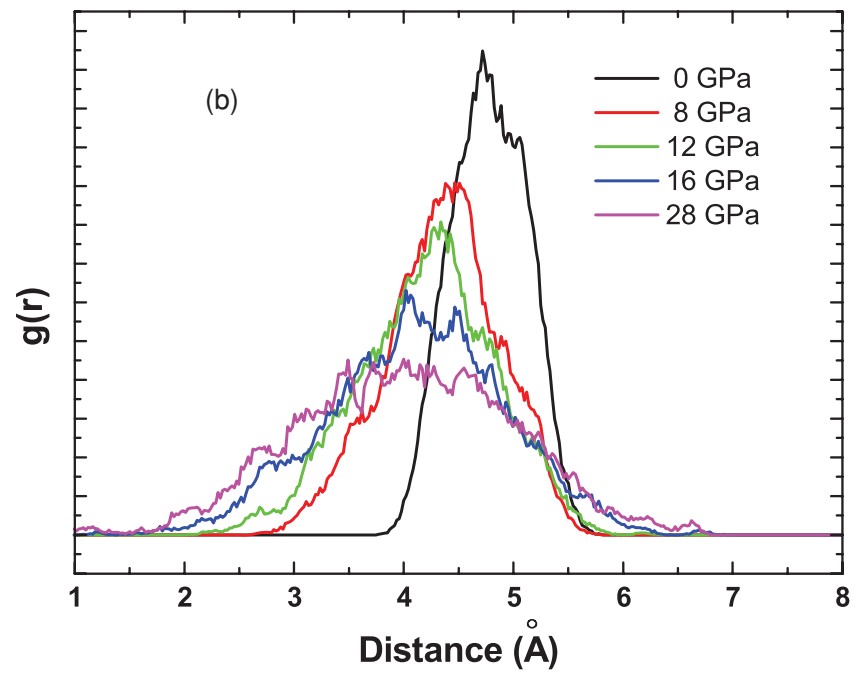

FIG. 8. Radial distribution functions with respect to the central point of the cages at five different pressures: for pentagonal dodecahedra $\left[5^{12}\right]$ cage (a) and tetrakaidecahedra $\left[5^{12} 6^{2}\right]$ cage (b).

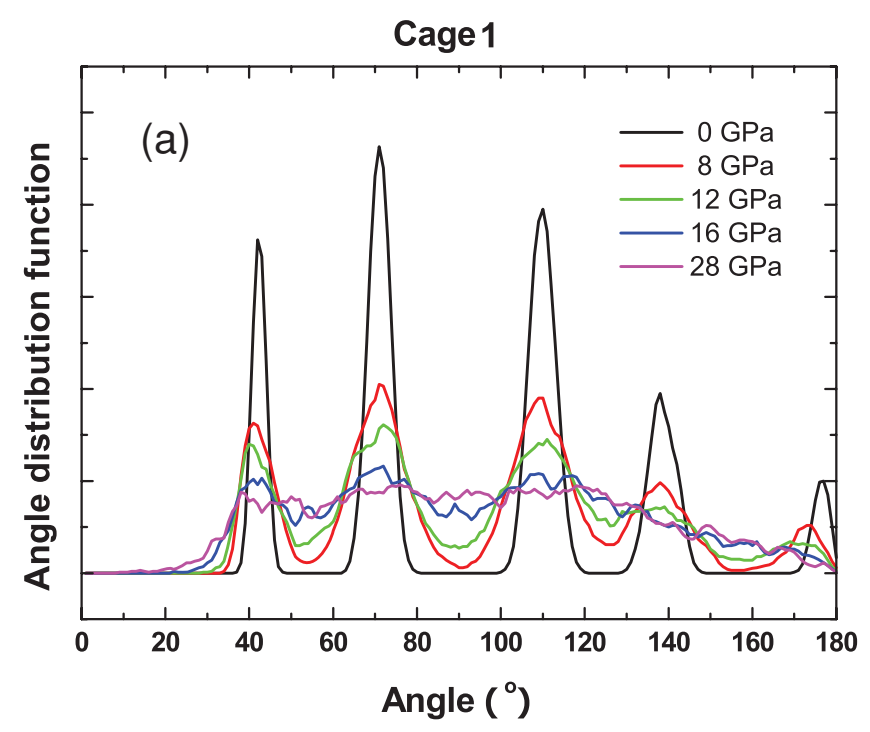

Cage 2

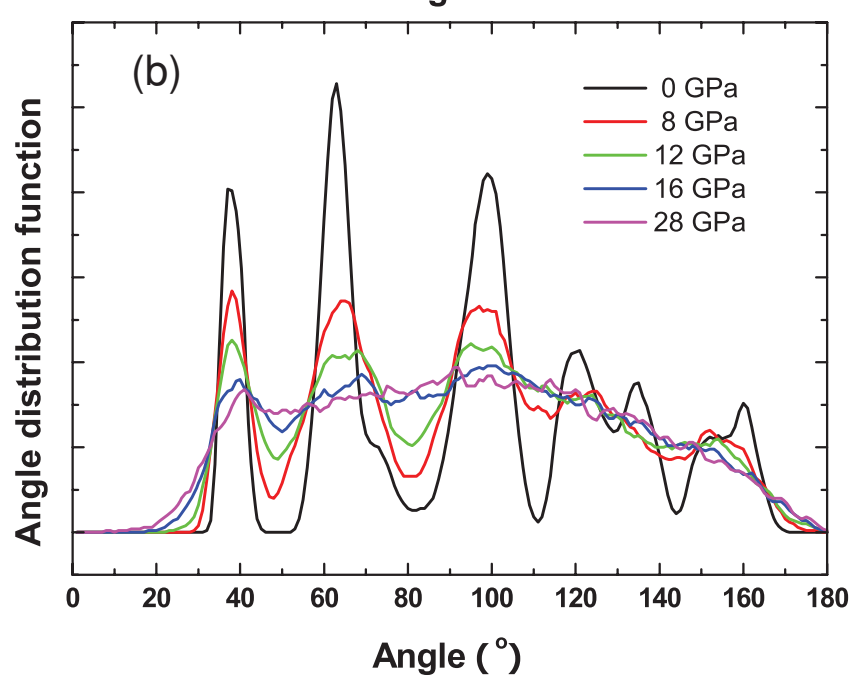

FIG. 9. Angle distributions with respect to the central point of the cages at five different pressures: for pentagonal dodecahedra $\left[5^{12}\right]$ cage (a) and tetrakaidecahedra $\left[5^{12} 6^{2}\right]$ cage (b).

the diffraction pattern is consistent with the experimental data. ${ }^{9} 10$ The relative intensity differs between different experimental reports. Our data show higher intensities in the second and third peaks (i.e., [202] and [222] assuming a tetragonal unit cell ${ }^{14}$ ) than those of Ref. 9 but similar to those of Ref. 10. The fact that the experimental data differ may result from the fact that the experimental specimens contain different degrees of disorder. With increasing pressure, we found that the strongest peak [423] and other peaks, shown in Fig. 6(b), decrease in intensity, which is in good agreement with experiments. ${ }^{9}$ At $8 \mathrm{GPa}$, the $\mathrm{x}$-ray structure factor is consistent with an amorphous state. As shown in Fig. 7, the $\mathrm{Si}-\mathrm{Si}$ pair distribution function is also flat at large distances, at 8 $\mathrm{GPa}$. The results of the radial distribution function analysis for the response of the cages to pressure are shown in Fig. 8. The cages compress mainly through a bending of the $\mathrm{Si}-\mathrm{O}-\mathrm{Si}$ angle and their distortion increases with pressure. We show the angle distribution functions between $\mathrm{Si}-$ center-Si, 
cage 1, $12 \mathrm{GPa}$
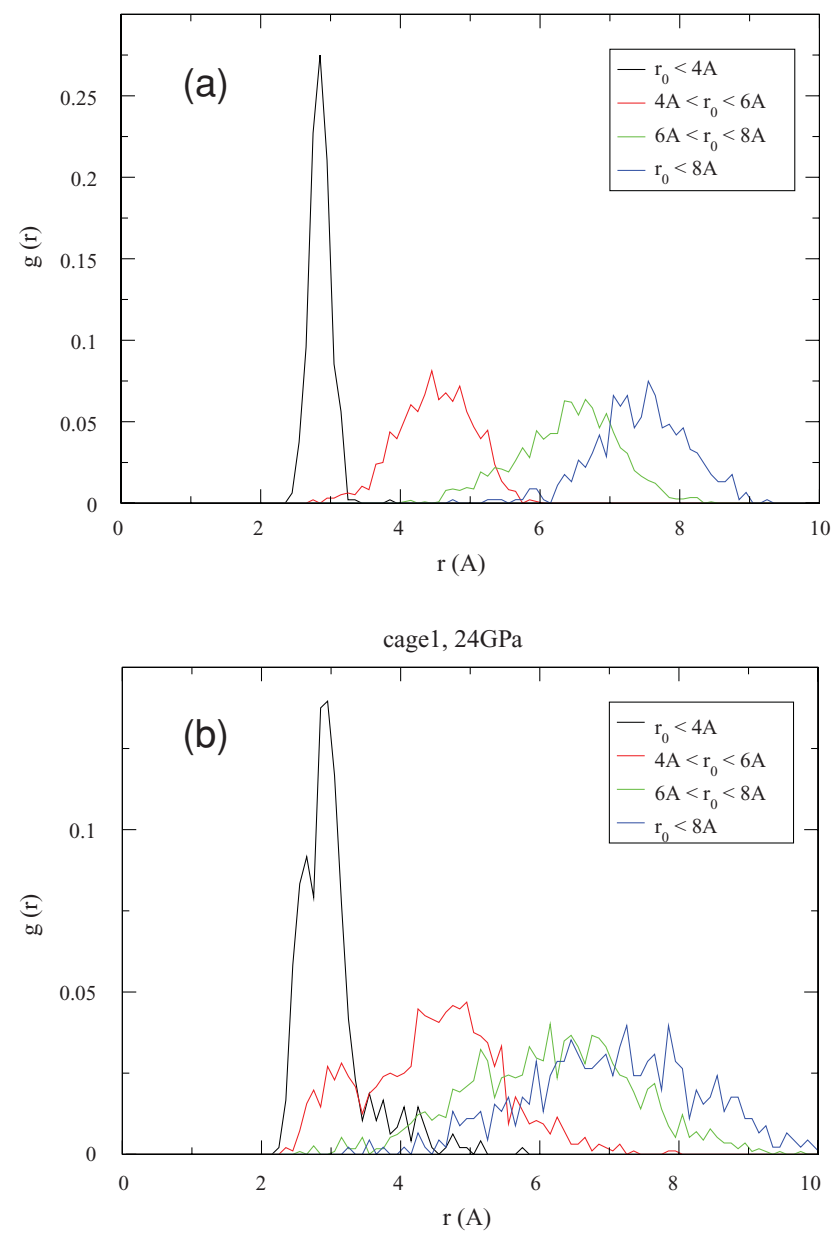

cage2, 12GPa

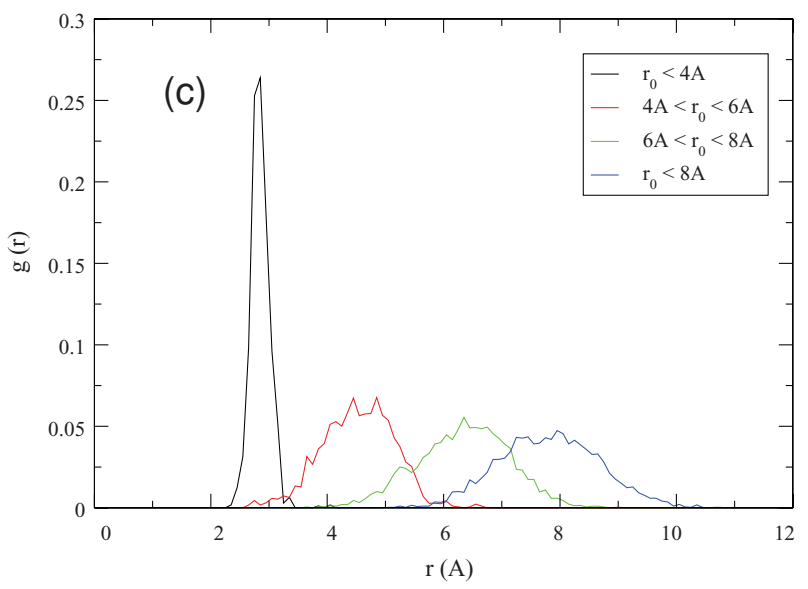

cage2, 24GPa

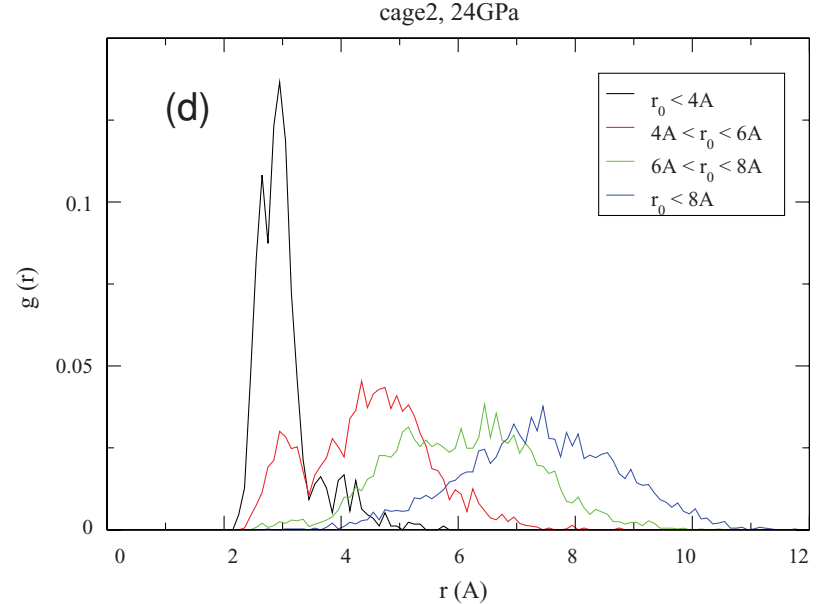

FIG. 10. Si-Si radial distribution functions on the surface of pentagonal dodecahedra $\left[5^{12}\right]$ at 12 (a) and $24 \mathrm{GPa}$ (b) and tetrakaidecahedra $\left[5^{12} 6^{2}\right]$ at 12 (c) and $24 \mathrm{GPa}(\mathrm{d})$. Pair distances divided into four groups based on their separation, $r_{0}$, at zero pressure.

where "center" is the center of the cages, in Fig. 9. The peak at $42^{\circ}$ corresponds to the nearest-neighbor $\mathrm{Si}$ atoms in cage 1 , the angle at $70^{\circ}$ corresponds to the next-nearest neighbors within the same pentagonal rings, and the angles at $110^{\circ}$, $138^{\circ}$, and $177^{\circ}$ correspond to the neighbors within the face pentagonal rings. The angle distribution in cage 2 can also be assigned to well defined cage elements. Under pressure, cage 2 loses the two large-angle features at $8 \mathrm{GPa}$ and three sharp peaks at $12 \mathrm{GPa}$, while cage 1 still contains all the features at $12 \mathrm{GPa}$. This suggests that larger cages are more easily distorted under pressure.

The addition of guest molecules can stabilize a clathrate lattice, acting as an organizing center for the formation of the lattice. ${ }^{25}$ The interactions between the guests and the host cages hold the cage in shape at much higher pressures than those of guest-free clathrates. Our simulations are for guestfree lattices, but we can explore this hypothesis by looking at how the structure of the cages changes at distances corresponding to the cage diameter with increasing pressure. For clarity, the $\mathrm{Si}-\mathrm{Si}$ pair separations between atoms on the surface of each cage were divided into four groups, based on their interatomic distance, $r_{0}$, at zero pressure: $r_{0}<4 \AA, 4 \AA<r_{0}$ $<6 \AA, 6 \AA<r_{0}<8 \AA$, and $r_{0}>8 \AA$. (The largest Si-Si separation at zero pressure is $9.1 \AA$ in cage 1 and
$10.7 \AA$ in cage 2.) The separations were tracked as pressure was increased, and Fig. 10 shows these distances at 12 and $24 \mathrm{GPa}$. At $12 \mathrm{GPa}$, the distortion of the cages has simply broadened the distribution of distances. At $24 \mathrm{GPa}$, however this broadening is much larger and there are clear signs of topological changes. In both cages, there are substantial peaks at the $\mathrm{Si}-\mathrm{O}-\mathrm{Si}$ bond length $(\sim 2.4 \AA)$ from pairs with $4 \AA<r_{0}<6 \AA$. There are also smaller contributions at this distance and substantial contributions at $\sim 4 \AA$ from atoms with $r_{0}>6 \AA$. This implies that pairs of silicon atoms which were at large separations at zero pressure are forming chemical bonds at $24 \mathrm{GPa}$, presumably in a $\mathrm{Si}-\mathrm{O}-\mathrm{Si}$ or longer chain, altering the topology and irreversibly amorphizing the structure. It also implies that this amorphization would not occur at this pressure in the presence of guest atoms. Guest atoms would physically obstruct the formation of $\mathrm{Si}-\mathrm{O}-\mathrm{Si}$ bonds starting from pairs of atoms separated by nearly the diameter of the whole cage and hence maintain the structure of the melanophlogite cages to higher pressures, as seen in Ref. 25 for clathrasils.

\section{The recovered sample}

The recovered sample shows densification above around $12 \mathrm{GPa}$ (Fig. 11), which suggests that the reduction of the 


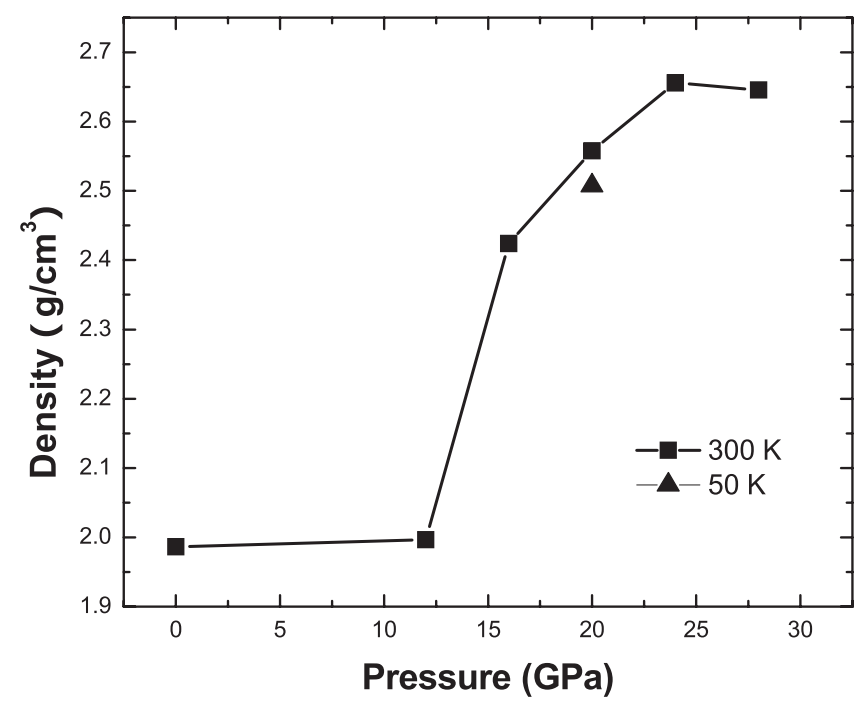

FIG. 11. Density of the recovered sample at $0 \mathrm{GPa}$, as a function of subjected peak pressure, for different starting samples.

Bragg peaks below $12 \mathrm{GPa}$ is not a consequence of bonding changes. This is confirmed by the analysis of the coordination changes and ring statistics (Figs. 12 and 13). The coordination increases only above $\sim 12 \mathrm{GPa}$, and as a consequence, the topology changes to generate a densified glass. The crystalline melanophlogite contains only five-membered, six-membered, and ten-membered rings. The topology of the recovered sample from $12 \mathrm{GPa}$ has two five-membered rings and one six-membered ring less than the starting crystal. The sample recovered from $16 \mathrm{GPa}$ shows some memory of the crystal according to the ring size distribution functions, which in this case contains a maximum for five-membered rings and a second peak for ten-membered rings. At higher pressure, the densification process saturates and generates a ring statistics similar to that of the densified glass. It is noteworthy that at higher subjected peak pressure, the densification is saturated the way pristine glass behaves as reported in Ref. 29 .

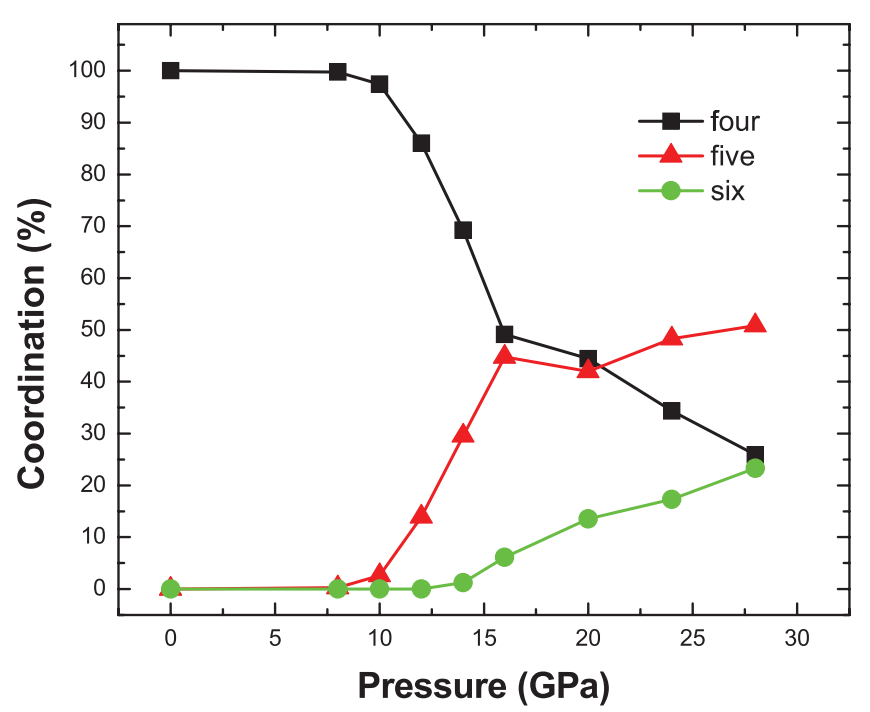

FIG. 12. Percentages of Si atoms coordinated four (squares), five (circles), and six (triangles), to oxygen, at different pressures.

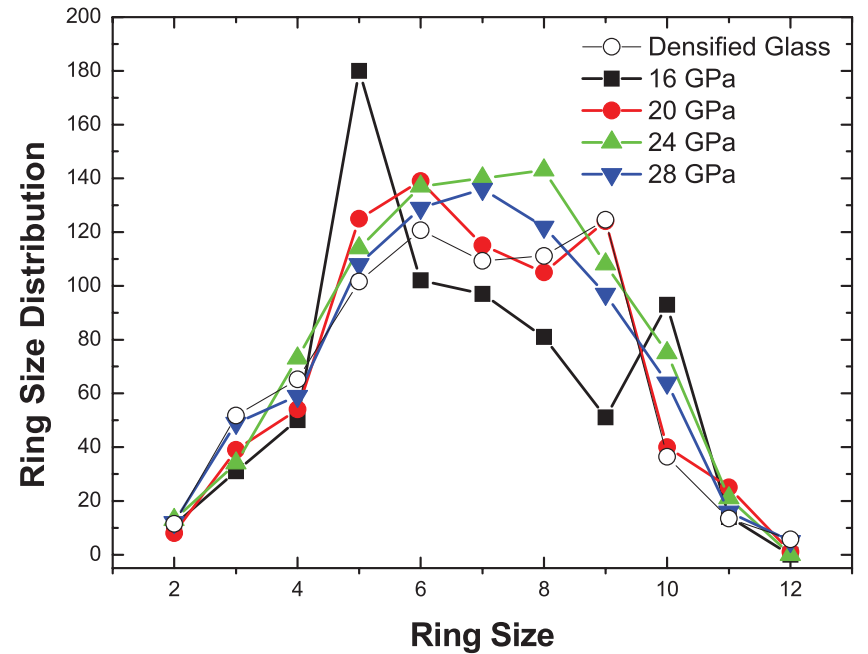

FIG. 13. Ring statistics at zero pressure for sample recovered from different peak pressures. The ring statistics of densified glass (Ref. 29) subjected to pressure $\sim 28 \mathrm{GPa}$ is also shown for comparison.

We remark that the increase of small-membered rings with peak pressure is consistent with Raman experimental data. ${ }^{36}$ A recent study ${ }^{8}$ of the pressure-induced amorphization of silicalite using RMC modeling has suggested the existence of a topologically ordered amorphous silica phase. We observed a topologically ordered amorphous structure at 8-12 GPa, but this structure reverts back to melanophlogite upon releasing pressure. Our recovered densified amorphous phase is topologically disordered. In other words, irreversible amorphization in melanophlogite is a consequence of the topological changes. We remark that the density of our topologically ordered amorphous phase at $8 \mathrm{GPa}$ coincides with the density of the densified amorphous phase at $0 \mathrm{GPa}$ and also with the density of densified silicalite $\left(2.7 \mathrm{~g} / \mathrm{cm}^{3}\right)$, so density alone cannot be used to discriminate between topologically distinct structures. Molecular dynamics simulations similar to the ones reported here for melanophlogite might be able to provide complementary information, with respect to RMC, on the structure of the densified amorphous phase obtained from silicalite.

\section{CONCLUSIONS}

To summarize, we have shown that the TangneyScandolo potential can reproduce the experimentally reported transition from a low-temperature distorted structure to a high-temperature cubic structure in melanophlogite. The distortion has been analyzed based on the structural changes of the individual cages. The simulation of high-pressure melanophlogite does not show a sharp volume collapse; instead, it gradually transforms into an amorphous phase. Below about $12 \mathrm{GPa}$, the transition is reversible: there is no significant change in the bonding or topology, as shown by the ring statistics distributions, and analysis of the coordination numbers and structure. Above this pressure, the cages deform sufficiently to alter the bonding and topology, and phases recovered from above this pressure are altered irreversibly. The densified amorphous phase at intermediate pressure 
contains some crystalline features as manifested by the ring statistics distributions. This connection between topological changes and permanent amorphization offers an alternative interpretation of the recent experimental results obtained on silicalite. $^{8}$

${ }^{1}$ O. Mishima, L. D. Calvert, and E. Whalley, Nature (London) 310, 393 (1984).

${ }^{2}$ R. J. Hemley, A. P. Jephcoat, H. K. Mao, L. C. Ming, and M. H. Manghnani, Nature (London) 334, 52 (1988).

${ }^{3}$ P. Richet and P. Gillet, Eur. J. Mineral. 9, 907 (1997).

${ }^{4}$ S. M. Sharma and S. K. Sikka, Prog. Mater. Sci. 40, 1 (1996).

${ }^{5}$ G. N. Greaves, F. Meneau, A. Sapelkin, L. M. Colyer, I. Ap Gwynnp, S. Wade, and G. Sakar, Nature Mater. 2, 622 (2003).

${ }^{6}$ G. N. Greaves, F. Meneau, O.Majerus, D. G. Jones, and J. Taylor, Science 308, 1299 (2005).

${ }^{7}$ I. Peral and J. Iniguez, Phys. Rev. Lett. 97, 225502 (2006).

${ }^{8}$ J. Haines, C. Levelut, A. Isambert, P. Hébert, S. Kohara, D. A. Keen, T. Hammouda, and D. Andrault, J. Am. Chem. Soc. 131, 12333 (2009).

${ }^{9}$ H. W. Xu, J. Z. Zhang, Y. S. Zhao, G. D. Guthrie, D. D. Hickmott, and A. Navrotsky, Am. Mineral. 92, 166 (2007).

${ }^{10}$ T. Yagi, E. Iida, H. Hirai, N. Miyajima, T. Kikegawa, and M. Bunno, Phys. Rev. B 75, 174115 (2007).

${ }^{11}$ A. L. Lasaulx, Neues Jahrb. Mineral. Monatsh. 1876, 250 (1876).

${ }^{12}$ B. J. Skinner and D. E. Appleman, Am. Mineral. 48, 854 (1963).

${ }^{13}$ B. Kamb, Science 148, 232 (1965).

${ }^{14}$ H. Gies, H. Gerke, and F. Liebau, Neues Jahrb. Mineral. Monatsh. 3, 119 (1982).

${ }^{15}$ L. Žák, Am. Mineral. 57, 779 (1972).

${ }^{16}$ H. Gies, Z. Kristallogr. 164, 247 (1983).
${ }^{17}$ C. A. Fyfe and H. Gies, J. Inclusion Phenom. Mol. Recognit. Chem. 8, 235 (1990).

${ }^{18}$ S. X. Liu, M. D. Welch, and J. Klinowski, J. Phys. Chem. B 101, 2811 (1997).

${ }^{19}$ T. Nakagawa, K. Kihara, and K. Harada, Am. Mineral. 86, 1506 (2001).

${ }^{20}$ A. Navrotsky, H. W. Xu, E. C. Moloy, and M. D. Welch, Am. Mineral. 88, 1612 (2003).

${ }^{21}$ T. Nakagawa, K. Kihara, and S. Fujinami, J. Miner. Pet. Sci. 100, 247 (2005).

${ }^{22}$ T. Nakagawa and K. Kihara, J. Miner. Pet. Sci. 101, 14 (2006).

${ }^{23}$ B. A. Kolesov and C. A. Geiger, Am. Mineral. 88, 1364 (2003).

${ }^{24}$ J. Kortus, G. Irmer, J. Monecke, and M. R. Pederson, Modell. Simul. Mater. Sci. Eng. 8, 403 (2000).

${ }^{25}$ J. S. Tse, D. D. Klug, J. A. Ripmeester, S. Desgreniers, and K. Lagerec, Nature (London) 369, 724 (1994).

${ }^{26}$ P. Tangney and S. Scandolo, J. Chem. Phys. 117, 8898 (2002).

${ }^{27}$ D. Herzbach, K. Binder, and M. H. Müser, J. Chem. Phys. 123, 124711 (2005).

${ }^{28}$ Y. F. Liang, C. R. Miranda, and S. Scandolo, J. Chem. Phys. 125, 194524 (2006).

${ }^{29}$ Y. F. Liang, C. R. Miranda, and S. Scandolo, Phys. Rev. B 75, 024205 (2007).

${ }^{30}$ Y. F. Liang, C. R. Miranda, and S. Scandolo, High Press. Res. 28, 35 (2008).

${ }^{31}$ Y. F. Liang, C. R. Miranda, and S. Scandolo, Phys. Rev. Lett. 99, 215504 (2007).

${ }^{32}$ A. M. Saitta and F. Datchi, Phys. Rev. E 67, 020201 (2003).

${ }^{33}$ A. M. Saitta, T. Strassle, G. Rousse, G. Hamel, S. Klotz, R. Nelmes, and J. S. Loveday, J. Chem. Phys. 1218430 (2004).

${ }^{34}$ A. M. Saitta, T. Strassle, and S. Klotz, J. Phys. Chem. B 1103595 (2006).

${ }^{35}$ L. P. Huang and J. Kieffer, Phys. Rev. Lett. 95, 215901 (2005).

${ }^{36}$ R. J. Hemley, H. K. Mao, P. M. Bell, and B. O. Mysen, Phys. Rev. Lett. 57, 747 (1986). 